\title{
Numerical modelling of the radiation efficiency of asymmetrical structures
}

\author{
J. Poblet-Puig ${ }^{\mathrm{a}, 1,2}$, A. Rodríguez-Ferran ${ }^{\mathrm{a}, *, 2}$, \\ C. Guigou-Carter ${ }^{b}$ and M. Villot ${ }^{b}$ \\ ${ }^{a}$ Laboratori de Càlcul Numèric \\ E.T.S. d'Enginyers de Camins, Canals i Ports de Barcelona \\ Campus Nord, Universitat Politècnica de Catalunya, Spain \\ ${ }^{\mathrm{b}}$ Centre Scientifique et Technique du Bâtiment \\ Département Acoustique et Éclairage \\ 24 Rue Joseph Fourier, 38400 Saint Martin d'Hères, France
}

\begin{abstract}
The radiation efficiency of a structural element is required by some models in order to predict its sound insulation. A common assumption is that the radiation on both sides of the element is the same. This is not true for asymmetrical structural elements like lightweight floors consisting of a beam-supported flat board. The radiation efficiency is larger on the beam side, because the beams act as exciters and increase the pressure level in the room. These different radiation efficiencies are calculated here for a two-dimensional cross-section by using finite elements and boundary elements. The obtained preliminary results illustrate that considering a single radiation efficiency can be a source of errors and that further investigation is required in order to improve predictions.
\end{abstract}

Key words: radiation efficiency, lightweight floor, finite element method PACS: 43.40.Rj 43.55.Ka

\footnotetext{
* Jordi Girona 1, Campus Nord, E-08034 Barcelona, Spain

Email addresses: jordi.poblet@upc.edu (J. Poblet-Puig), antonio.rodriguez-ferran@upc.edu (A. Rodríguez-Ferran), catherine.guigou@cstb.fr (C. Guigou-Carter), michel.villot@cstb.fr (M. Villot).

1 Fons Social Europeu (2003 FI 00652)

2 Ministerio de Educación y Ciencia (BIA2007-66965, DPI2007-62395). Research Fund for Coal and Steel (RFSR-CT-2003-00025).
} 


\section{Introduction}

The radiation of sound from a vibrating elastic solid immersed in an acoustic fluid is a common problem in acoustic engineering. The most relevant output of interest is the radiation efficiency $\sigma$, defined as

$$
\sigma=\frac{\mathcal{P}_{\text {rad }}}{\rho_{0} c S<\widetilde{V_{n}^{2}}>} \quad \text { with } \quad \mathcal{P}_{\text {rad }}=\oint \boldsymbol{I} \cdot n d S
$$

where $\mathcal{P}_{\text {rad }}$ is the acoustic power flow through a closed, arbitrary surface radiated by the vibrating solid, $c$ the speed of sound, $\rho_{0}$ the fluid density, $I$ the acoustic intensity, $S$ the surface of the radiating body and $V_{n}$ the normal velocity of the vibrating surface, averaged in time $(\widetilde{\bullet})$ and in space $(<\bullet>)$.

An important issue in building acoustics is whether the radiation efficiency of a wall or a floor is the same for both faces. The structural elements are often not symmetric (e.g. beam-supported flat board). The radiation efficiency is an input parameter of SEA models and some flanking transmission models [1,2]. A common assumption is that the radiation efficiency is the same for both sides. However, for the case of lightweight structures it may be important to use two different values depending on the radiation side. This is suggested in $[3,4]$ and corroborated here on the basis of a numerical simulation.

In Section 2, the problem is studied at the cross-section level. Two-dimensional FEM and BEM models are used in order to calculate the radiation efficiency of a flat board. In Section 3 the radiation of a floor with beams is considered.

\section{A numerical model for the calculation of the radiation efficiency}

In the examples presented here, finite domains are considered. The radiation efficiency, Eq. (1), is computed from outputs of the numerical model. The averaged velocity $\left\langle\widetilde{V_{n}^{2}}>\right.$ can be obtained by averaging the vibration field over a structure. In order to obtain the radiated power two assumptions are made: $i)$ the pressure field in the room is diffuse; ii) the absorption of the room can be calculated by means of the admittance in the absorbing contours.

By means of simplified wave-based models $[5,6]$, a relation between the averaged absorption coefficient $\alpha_{i}$ and the impedance of absorbing boundaries can be established. Then the total absorbed acoustic power can be calculated as

$$
\mathcal{P}_{a b s}=\sum_{i} S_{i} I_{i n c} \alpha_{i}
$$

where $S_{i}$ is the surface of each absorbing boundary and $\alpha_{i}$ its absorption coefficient. $I_{i n c}$ is the incident acoustic intensity in the room contours.

The first assumption is not satisfied in the low frequency range where the 


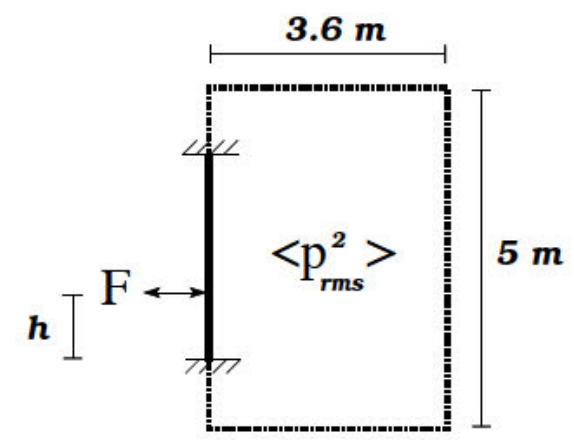

Fig. 1. Radiation of sound of a mechanically excited leave.

pressure distribution follows the shape of the nearest eigenmode. However, the modal density rapidly increases with the room size. In that case the average of the pressure fields obtained for several consecutive frequencies is a reverberant field, and the incident acoustic intensity in the room contours is

$$
I_{i n c}=\frac{<p_{r m s}^{2}>}{4 \rho_{0} c}
$$

where $p_{r m s}=|p| / \sqrt{2}$ is the root mean square pressure and $p$ the phasor of acoustic pressure.

Since energy losses are only caused by acoustic absorption, this absorbed power is the power radiated by the vibrating structure, $\mathcal{P}_{\text {rad }}=\mathcal{P}_{\text {abs }}$. The radiation efficiency of the structure is calculated by substitution of the known values $\mathcal{P}_{\text {rad }}$ and $<\widetilde{V_{n}^{2}}>$ in Eq. (1).

The first example deals with a wood panel that is excited by means of a point force and radiates sound into a rectangular room. The dimensions of the room are $3.6 \mathrm{~m} \times 5 \mathrm{~m}$. Seven positions of the point force are considered in order to obtain averaged outputs: $h=0.25,0.5,1,1.5,2,2.5$ and $2.75 \mathrm{~m}$, see Fig. 1 . The analyses in this work have been focused on mechanical excitation but can be easily extended to acoustic excitation. The mechanical and geometrical properties are summarised in Table 1 . The calculated radiation efficiency is plotted in Fig. 2. It has been compared with the analytical expressions of the radiation efficiency for a single panel that can be found in [7],

$$
\sigma=\left\{\begin{array}{cl}
\frac{c^{2}}{S f_{c}^{2}} g_{1}(\alpha)+\frac{c P}{S f_{c}} g_{2}(\alpha) & \text { if } f<f_{c} \\
\sqrt{\omega \ell_{x} / c}\left(0.5-0.15 \frac{\ell_{x}}{\ell_{y}}\right) & \text { if } f=f_{c} \\
1 / \sqrt{1-f_{c} / f} & \text { if } f>f_{c}
\end{array}\right.
$$

where $P=2\left(\ell_{x}+\ell_{y}\right)$ is the perimeter of the plate and $S=\ell_{x} \times \ell_{y}$ is its surface $\left(\ell_{x}=1 \mathrm{~m}, \ell_{y}=3 \mathrm{~m}\right) . \omega=2 \pi f$ is the angular frequency of the problem, $f_{c}$ is the critical frequency of an infinite the plate $\left(f_{c}=c^{2} \sqrt{\rho_{\text {solid }} / E} / 1.8 t\right)$ and 
Table 1

Material and geometrical properties for the example of a single vibrating element.

\begin{tabular}{lll} 
Meaning & Symbol & Value \\
\hline Young's modulus & $E$ & $10^{10} \mathrm{~N} / \mathrm{m}^{2}$ \\
Poisson's ratio & $\nu$ & 0.25 \\
Solid density & $\rho_{\text {solid }}$ & $400 \mathrm{~kg} / \mathrm{m}^{3}$ \\
Thickness & $t$ & $0.02 \mathrm{~m}$ \\
Bending inertia & $I$ & $6.67 \cdot 10^{-7} \mathrm{~m}^{4} / \mathrm{m}$ \\
Cross section area & $A$ & $0.02 \mathrm{~m}^{2} / \mathrm{m}$ \\
Wall length & $\ell$ & $3 \mathrm{~m}$ \\
\hline
\end{tabular}

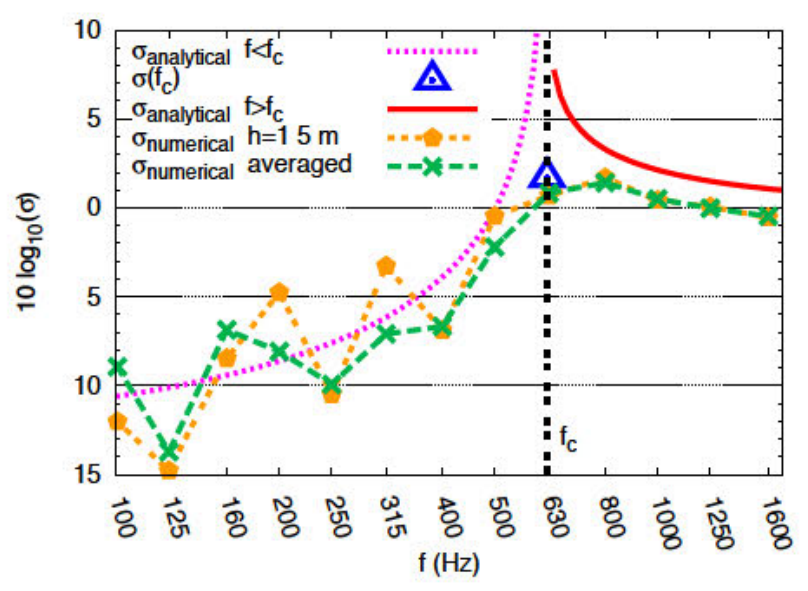

Fig. 2. Radiation efficiency of case of Fig. 1.

$\alpha=\sqrt{f / f_{c}}$. The functions $g_{1}$ and $g_{2}$ are

$$
\begin{gathered}
g_{1}(\alpha)=\left\{\begin{array}{cc}
\frac{8}{\pi^{4}} \frac{1-2 \alpha^{2}}{\alpha \sqrt{1-\alpha^{2}}} & \text { if } f \leq f_{c} / 2 \\
0 & \text { if } f>f_{c} / 2
\end{array}\right. \\
g_{2}(\alpha)=\frac{1}{4 \pi^{2}}\left(\frac{\left(1-\alpha^{2}\right) \ln \left(\frac{1+\alpha}{1-\alpha}\right)+2 \alpha}{\left(1-\alpha^{2}\right)^{3 / 2}}\right)
\end{gathered}
$$

The smaller values of radiation are found for low frequencies, where oscillations caused by the modal behaviour of the structure and the rooms occur. At the critical frequency $f_{c}$ the wave length of acoustic and vibration waves is the same and the radiation efficiency reaches its maximum value. For high frequencies, as expected, the radiation efficiency tends to a constant value. Two different numerical radiation efficiency curves are shown in Fig. 2. One of them is the case with only a point load $(h=1.5 \mathrm{~m})$ while the other corresponds 
Table 2

Eigenfrequency $f_{n}$ and geometrical coincident frequency $f_{g c}$ of structural modes.

\begin{tabular}{c|cccccccc}
$n$ & 1 & $\ldots$ & 10 & 11 & 12 & 13 & $\ldots$ & 16 \\
\hline$f_{g c}$ & 56.67 & $\ldots$ & 566.67 & $\mathbf{6 2 3 . 3 3}$ & $\mathbf{6 8 0}$ & 736.67 & $\ldots$ & 906.67 \\
$f_{n}$ & 5.04 & $\ldots$ & 504.76 & $\mathbf{6 1 0 . 9 9}$ & $\mathbf{7 2 7 . 4 4}$ & 854.12 & $\ldots$ & 1295.89 \\
\hline
\end{tabular}

to the average of the seven load positions. Differences are more relevant in the low frequency range where the structural vibration depends on the excited modes. On the contrary, very small differences are found for mid and high frequencies.

The radiation efficiency is high when the wave number of the structural vibrations and the pressure wave are similar [8]. The wave number for the vibration mode associated with the eigenfrequency

$$
f_{n}=\frac{1}{2 \pi} \sqrt{\frac{E I}{\rho_{\text {solid }}\left(A-I\left(\frac{n \pi}{\ell}\right)^{2}\right)}}\left(\frac{n \pi}{\ell}\right)^{2}
$$

is $k_{n}=n \pi / \ell$. The wave number of pressure waves is $k=\omega / c$. Thus, if we impose both wave numbers to be the same, $k=k_{n}$, we can define a geometrical coincidence frequency $f_{g c}$. For the case of the beam and considering parallel acoustic waves, it can be expressed as $f_{g c}=k_{n} c / 2 \pi$ (this value is different if other directions of acoustic waves are considered).

The maximum radiation is reached when the two frequencies $f_{n}$ and $f_{g c}$ are similar. In this situation, a frequency $f \approx f_{n} \approx f_{g c}$ excites the eigenmodes of the structure that can radiate more sound. In Table 2 the structural eigenfrequency $f_{n}$ and the geometrical coincidence frequency $f_{g c}$ for some structural modes are shown. We can see how this critical frequency of approximately $630 \mathrm{~Hz}$ is found between modes 11 and 12 . In the numerical experiment, the maximum radiation efficiency is also found in this frequency range (see Fig. 2).

\section{The role of beams in the radiation of a surface}

The second example deals with the beam-supported flat board shown in Fig. 3(a). The goal of the analysis is to assess the difference in radiation depending on the side of the floor. Since we are interested in taking into account the shape of the beams, the acoustic domains are not rectangular. For this reason, models that use analytical modal analysis in the acoustic domains in order to reduce the computational costs (e.g. [9-11]) cannot be used. The floor is composed of a wood board and I-shaped beams (73 $\mathrm{mm}$ in width and $140 \mathrm{~mm}$ in height). The separation between steel beams is $350 \mathrm{~mm}$. The rooms dimensions are $4 \mathrm{~m} \times 3.6 \mathrm{~m}$. The structural cross-section is modelled 


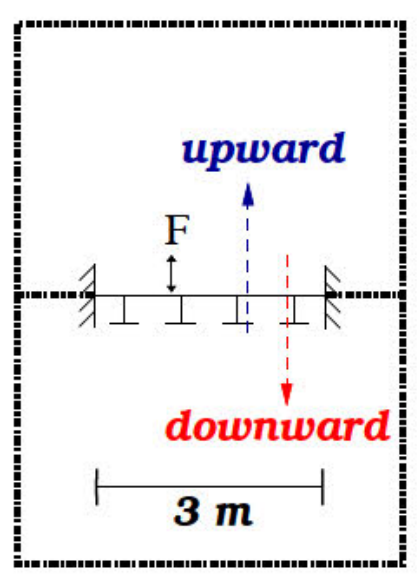

(a)

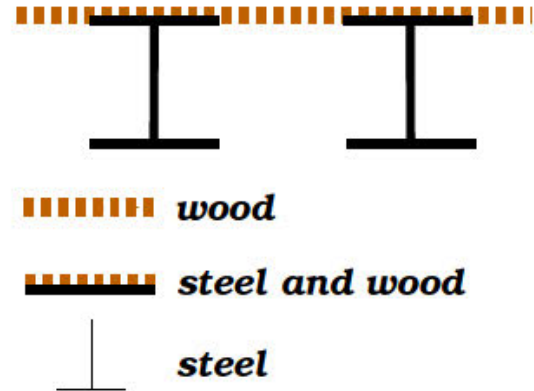

(b)

Fig. 3. (a) Sketch of the radiation of an asymmetric floor into two rooms; (b) detail of the three zones with different material and geometrical properties

by means of beam finite elements. As seen in Fig. 3(b) and in Table 3, three different regions have been considered: (1) wood board, (2) steel stud and (3) wood board and steel stud overlapped. Homogenised geometrical and mechanical properties have been used in the latter case (they are indicated with an asterisk). Mixed cross-sections are characterised by means of a unique set of parameters taking into account the different mechanical properties of steel and wood. The floor is excited by a point force located on the upper flange of the second stud (in view of the results of Fig. 2, only one force location is considered for this preliminary analysis).

As a reference, the board without beams is also analysed. In both cases (i.e. with and without beams), the surface considered to calculate the radiation efficiency is $S=3 \mathrm{~m}$ (i.e. the width of the board, see Fig. 3(a)).

The results are presented in Fig. 5. The differences in $\sigma$ are significant. The radiation efficiency is larger for the side with steel beams. The radiation upwards (planar face) is very similar to that of the board without studs.

The averaged velocity levels of the upper and lower faces are very similar. For the lower face, this average takes into account both the board and the beams. On the contrary, the pressure in the lower room is higher than in the upper room, see Fig. 5(b). Consequently the radiated power is also higher downwards than upwards, see Eqs. (2) and (3). This causes the differences in the radiation efficiency.

The steel profiles act as exciters of the acoustic fluid in the room below, see Fig. 4, so it is reasonable that the sound level there is higher. The same tendency is also observed in laboratory measurements [12]. This effect can only be captured by models taking into account the geometry of the floor. 
Table 3

Geometrical and mechanical properties for the beam supported flat board.

\begin{tabular}{llll}
\multicolumn{1}{c}{ Physical region: } & & $\begin{array}{l}\text { Wood plate } \\
\text { Meaning }\end{array}$ & Steel section \\
Vambol & Value & Value \\
\hline Young's modulus & $E$ & $10^{10} \mathrm{~N} / \mathrm{m}^{2}$ & $2.0601 \cdot 10^{11} \mathrm{~N} / \mathrm{m}^{2}$ \\
Hysteretic damping coefficient & $\eta$ & 0.05 & 0.05 \\
Density & $\rho_{\text {solid }}$ & $400 \mathrm{~kg} / \mathrm{m}^{3}$ & $7500 \mathrm{~kg} / \mathrm{m}^{3}$ \\
Thickness & $t$ & $0.04 \mathrm{~m}$ & $0.01 \mathrm{~m}$ \\
\hline
\end{tabular}

Physical region: wood and steel

\begin{tabular}{lll} 
Meaning & Symbol & Value \\
\hline Young's modulus & $E^{*}$ & $2.0601 \cdot 10^{11} \mathrm{~N} / \mathrm{m}^{2}$ \\
Hysteretic damping coefficient & $\eta^{*}$ & 0.05 \\
Density & $\rho_{\text {solid }}^{*}$ & $7620.39 \mathrm{~kg} / \mathrm{m}^{3}$ \\
Equivalent thickness & $t^{*}$ & $1.194 \cdot 10^{-2} \mathrm{~m}$ \\
Equivalent inertia per unit width & $I^{*}$ & $1.358 \cdot 10^{-6} \mathrm{~m}^{3}$ \\
\hline
\end{tabular}

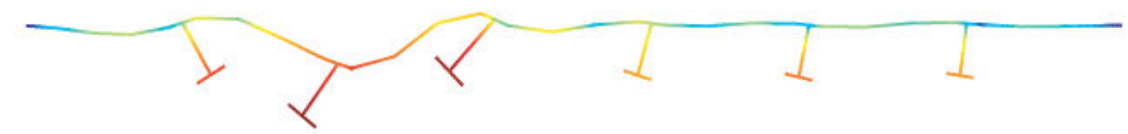

Fig. 4. Vibration of the floor with steel beams at $150 \mathrm{~Hz}$.

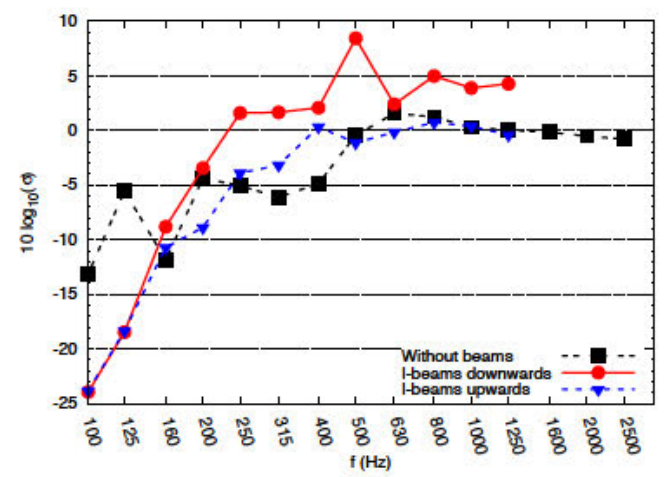

(a)

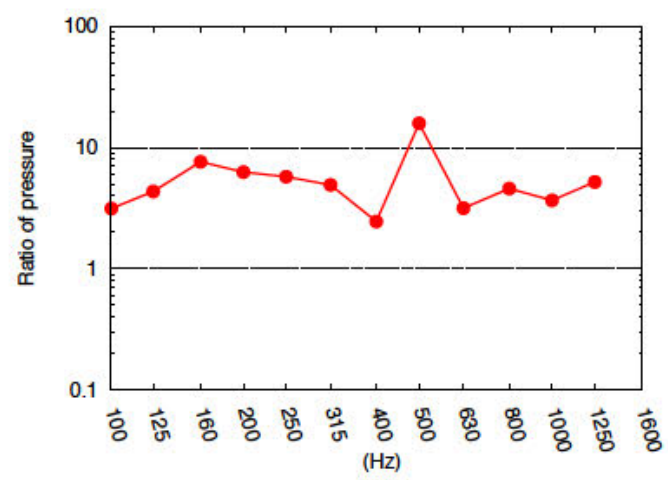

(b)

Fig. 5. Results for the example in Fig. 3(a): (a) radiation efficiency of a floor depending on the side; (b) ratio of pressures $\left.\left.<p_{\text {rms }}^{2}\right\rangle_{\text {downwards }} /<p_{\text {rms }}^{2}\right\rangle_{\text {upwards }}$.

\section{Concluding remarks}

The effect of steel beams in the radiation efficiency of a wood board has been studied. A two-dimensional and numerical-based model that describes the actual geometry at cross-section level has been used. The calculated differences in the radiation efficiency between plane and irregularly shaped (due to the studs) faces of the floor are around $4 \mathrm{~dB}$.

The obtained results suggest that considering the exact geometry can be relevant for the radiation efficiency of floors. For some construction elements, 
considering only the mass and thickness is not enough in order to perform accurate predictions of sound transmission.

The modelling of flanking transmissions is a typical case where it is often assumed that the radiation of sound is equal for both faces of a floor. For instance, the European regulation EN12354 [13] makes this assumption. This regulation applies to heavy structures, not to lightweight structures. However, [3] and [4] suggest that the extension of EN12354 to lightweight structures should rely on the use of adequate values of the radiation efficiency. The results presented here illustrate that the radiation efficiency can be different for the two faces of an asymmetrical structure.

\section{References}

[1] E. Gerretsen, Calculation of the sound transmission between dwellings by partitions and flanking structures, Appl. Acoust. 12 (6) (1979) 413-433.

[2] E. Gerretsen, Calculation of airborne and impact sound insulation beteen dwellings, Appl. Acoust. 19 (4) (1986) 245-264.

[3] T. Nightingale, Application of the CEN draft building acoustics prediction model to a lightweight double leaf construction, Appl. Acoust. 46 (3) (1995) 265-284.

[4] C. Guigou-Carter, M. Villot, R. Wetta, Prediction method adapted to wood frame lightweight constructions, Building Acoustics 13 (3) (2006) 173-188.

[5] A. Pierce, Acoustics. An introduction to its physical principles and aplications., McGraw Hill, New York, 1981.

[6] L. Bell, D. Bell, Industrial noise control, Dekker, New York, 1993.

[7] G. Maidanik, Response of ribbed panels to reverberant acoustic fields, J. Acoust. Soc. Am. 34 (6) (1962) 809-826.

[8] C. Wallace, Radiation resistance of a rectangular panel, J. Acoust. Soc. Am. 51 (3) (1970) 946-952.

[9] L. Gagliardini, J. Roland, J. Guyader, The use of a functional basis to calculate acoustic transmission between rooms, J. Sound Vibr. 145 (3) (1991) 457-478.

[10] J. Brunskog, P. Davidsson, Sound transmission of structures. A finite element approach with simplified room description, Acta Acust. United Acust. 90 (5) (2004) 847-857.

[11] P. Jean, J. Rondeau, A simple decoupled modal calculation of sound transmission between volumes, Acta Acust. United Acust. 88 (2002) 924-933.

[12] M. Villot, C. Guigou-Carter, Measurement methods adapted to wood frame lightweight constructions, Building Acoustics 13 (3) (2006) 189-198.

[13] EN-12354, Building Acoustics: Estimation of the acoustic performance of buildings from the performance of elements, Tech. Rep. 1-4 (1999-2000). 